\title{
Segmentation of Non-viable Myocardium in Delayed Enhancement Magnetic Resonance Images
}

Arunark Kolipaka

Cleveland Clinic Foundation

George P. Chatzimavroudis

Cleveland State University

Richard D. White

Cleveland Clinic Foundation

Thomas P. O'Donnell

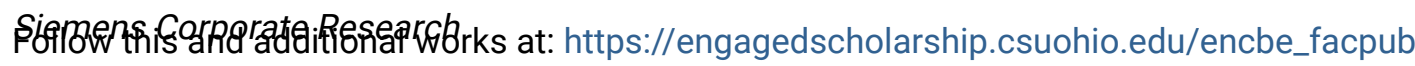

Irebotpdf the Betseraging and Biomedical Optics Commons

Glevelard clinice Foundatipn work benefit you? Let us know!

Publisher's Statement

The final publication is available at Springer via http://dx.doi.org/10.1007/s10554-004-5806-z

\section{Original Citation}

Kolipaka A, Chatzimavroudis GP, White RD, O'Donnell TP, Setser RM. Segmentation of non-viable myocardium in delayed enhancement magnetic resonance images. The International Journal of Cardiovascular Imaging. 2005;21:303-311.

Repository Citation

Kolipaka, Arunark; Chatzimavroudis, George P.; White, Richard D.; O’Donnell, Thomas P.; and Setser, Randolph M., "Segmentation of Non-viable Myocardium in Delayed Enhancement Magnetic Resonance Images" (2005). Chemical \& Biomedical Engineering Faculty Publications. 109.

https://engagedscholarship.csuohio.edu/encbe_facpub/109

This Article is brought to you for free and open access by the Chemical \& Biomedical Engineering Department at EngagedScholarship@CSU. It has been accepted for inclusion in Chemical \& Biomedical Engineering Faculty Publications by an authorized administrator of EngagedScholarship@CSU. For more information, please contact library.es@csuohio.edu. 


\title{
Segmentation of non-viable myocardium in delayed enhancement magnetic resonance images
}

\author{
Arunark Kolipaka ${ }^{1,2}$, George P. Chatzimavroudis ${ }^{1,2}$, Richard D. White ${ }^{1}$, Thomas P. \\ $\mathrm{O}^{\prime}$ Donnell ${ }^{3}$ \& Randolph M. Setser ${ }^{1,2}$ \\ ${ }^{1}$ Section of Cardiovascular Imaging, Division of Radiology, The Cleveland Clinic Foundation, Cleveland, \\ Ohio, USA; ${ }^{2}$ Department of Chemical and Biomedical Engineering, Cleveland State University, Cleveland, \\ Ohio, USA; ${ }^{3}$ Siemens Corporate Research, Princeton, New Jersey, USA
}

Key words: delayed enhancement, ischemic heart disease, magnetic resonance imaging, myocardial viability

\begin{abstract}
Purpose: To evaluate six algorithms for segmenting non-viable left ventricular (LV) myocardium in delayed enhancement (DE) magnetic resonance imaging (MRI). Methods: Twenty-three patients with known chronic ischemic heart disease underwent DE-MRI. DE images were first manually thresholded using an interactive region-filling tool to isolate non-viable myocardium. Then, six thresholding algorithms, based on the image intensity characteristics of either LV blood pool (BP), viable LV myocardium, or both, were applied to each image. For the Mean-2SD $\mathrm{BP}_{\mathrm{BP}}$ algorithm, thresholds were equal to the mean $\mathrm{BP}$ intensity minus twice its standard deviation. For the Mean $+2 \mathrm{SD}_{\mathrm{Semi}}$, Mean $+3 \mathrm{SD}_{\mathrm{Semi}}$, Mean $+2 \mathrm{SD}_{\mathrm{Aut}}$, and Mean $+3 \mathrm{SD}_{\text {Auto }}$ algorithms, thresholds equaled the mean intensity of viable myocardium plus twice (or thrice, as denoted by the name) the standard deviation of intensity (subscripts denote how these values were determined: automatic or semi-automatic). For the Minimum Intensity algorithm, the threshold equaled the minimum intensity between the BP and LV myocardium mean intensities. Percent Scar was defined as the ratio of non-viable to total myocardial pixels in each image. Agreement between each algorithm and manual thresholding was assessed using Bland-Altman analysis. Results: Mean Percent Scar was $25 \pm 16 \%$ by manual thresholding. Five of the six algorithms demonstrated mean bias within $\pm 3 \%$ (all except Mean $+2 \mathrm{SD}_{\text {Auto }}$ ); however, limits of agreement (LoA) were large in general (range 12-36\%). The best overall agreement was demonstrated by the Mean $+2 \mathrm{SD}_{\mathrm{Semi}}$ (bias, $0 \%$; LoA, $12 \%$ ) and Mean + $3 \mathrm{SD}_{\text {Semi }}$ (bias, $-3 \%$; LoA, $14 \%$ ) algorithms. Conclusion: On average, five of the six algorithms proved satisfactory for clinical implementation; however, in some images, manual correction of automatic results was necessary.

\section{Introduction}

Recovery of cardiac function after coronary revascularization depends upon the relative contributions of viable and non-viable myocardium within the left ventricle (LV) [1-3]. Delayed enhancement (DE) magnetic resonance imaging (MRI) can be used to non-invasively differentiate

viable from non-viable myocardium within the LV in patients [3-6], and has a spatial resolution superior to alternative non-invasive tests of viability [1]. Furthermore, animal models have shown that the area of hyperenhancement on DE-MRI images agrees closely with the location and extent of non-viable tissue demonstrated by post-mortem histologic staining of the myocardium $[7,8]$.
\end{abstract}


Previous studies using DE-MRI have typically relied on either visual inspection of images with a semi-quantitative analysis [3-6], or manually drawing contours around non-viable tissue $[9,10]$, which can be time prohibitive. Semi-automatic segmentation techniques, based on the image intensity characteristics of viable myocardium, have been used extensively [7, 8, 11-14], but have not been validated previously in the literature. However, given the marked contrast between viable and non-viable tissue in DE images, with an almost fivefold intensity difference between regions $[7,15]$, near-automatic segmentation of non-viable tissue may be possible.

Therefore, the objectives of this study were to develop and validate new methodology for automatic (or semi-automatic) segmentation of DE-MRI images, as well as to provide validation for previously introduced techniques. All methods were based on features of DE-MRI image histograms derived from the LV myocardium, the LV blood pool, or both combined.

\section{Methods}

All imaging studies were clinically indicated. Further image analysis was approved by the local Institutional Review Board with a waiver of individual consent. The study population consisted of 23 patients $[20$ male $/ 3$ female, age $59 \pm 13$ years) with known chronic ischemic heart disease referred for MRI assessment of myocardial viability.

\section{Imaging procedure}

Imaging was performed using a $1.5 \mathrm{~T}$ MRI scanner (Sonata, Siemens Medical Solutions, Erlangen, Germany). In each patient, short-axis DE-MRI was performed at the basal, middle, and apical thirds of the LV chamber, approximately $20 \mathrm{~min}$ after intravenous injection of $0.2 \mathrm{mmol} / \mathrm{kg}$ Gadolinium-DTPA (Magnevist, Berlex Laboratories, Wayne, NJ).

Fourteen of 23 patients $(61 \%)$ were imaged using an inversion recovery TurboFLASH pulse sequence (TE $4 \mathrm{~ms}$, TR $8 \mathrm{~ms}$, flip angle $30^{\circ}, 23$ lines acquired every other RR-interval, slice thickness 8-10 mm, FOV $300-360 \mathrm{~mm}$, RFOV $80-100 \%$, NSA 1 , initial matrix $256^{2}$ ) [15], with the inversion time (TI) optimized (TI 190-470 ms) to null the signal of viable myocardium in each patient (i.e. viable myocardium appears dark). This sequence is referred to as IR in this study.

In 9 of 23 patients (39\%), images were acquired using an IR sequence with phase sensitive reconstruction [16], which renders the images insensitive to TI; this version of the sequence is referred to as PS in this study. Acquisition parameters were identical to the sequence described above, except that a nominal TI of $250 \mathrm{~ms}$ was used for each patient. Briefly, with this technique a proton-density-weighted reference image (flip angle $5^{\circ}$ ) was acquired every other RR-interval which serves as both a phase map to preserve signal polarity and a coil sensitivity map to improve image contrast.

With either sequence, images were acquired during repetitive 10-15 s breath-holds, depending on the heart rate. In addition to short-axis imaging, DE imaging was performed in long-axis views including vertical (2-chamber), LV outflow tract (3-chamber) and horizontal (4-chamber) orientations.

\section{Image segmentation}

Epicardial and endocardial contours were drawn in each short-axis image using cardiovascular image analysis software (Argus, Siemens Medical Solutions, Erlangen, Germany). In regions of low blood-myocardial contrast (e.g. subendocardial infarcts), corresponding short-axis TrueFISP cine images were used to assist the user in drawing contours.

The extent of non-viable myocardium in each segmented image was determined using a manual thresholding technique as well as six automatic or semi-automatic thresholding algorithms, each of which resulted in a separate threshold value for each image. Each of these seven techniques (including manual thresholding) is described in more detail below.

Manual thresholding was performed using prototype analysis software based on the Argus package. With this software, users employ a suite of tools including an interactive thresholder and 
paint/erase brushes to label non-viable tissues, as described previously [10].

The six remaining thresholding algorithms were based on either image intensity characteristics of LV blood pool (Mean-2SD ${ }_{\mathrm{BP}}$ algorithm), image intensity characteristics of viable LV myocardium $\left(\right.$ Mean $+2 \mathrm{SD}_{\text {Semi }}, \quad$ Mean $+3 \mathrm{SD}_{\text {Semi }}, \quad$ Mean + $2 \mathrm{SD}_{\text {Auto }}$ and Mean $+3 \mathrm{SD}_{\text {Auto }}$ algorithms), or image intensity characteristics of both LV blood pool and myocardium (Minimum Intensity algorithm).

For the Mean-2SD ${ }_{\mathrm{BP}}$ algorithm, a region of interest (ROI) was automatically selected within the LV blood pool using the LV endocardial contour as a template. However, papillary muscles were excluded by confining the ROI to a semicircular region in the septal half of the blood pool (Figure 1). The threshold was defined as two times the standard deviation $(2 * \mathrm{SD})$ below the mean intensity within the ROI.

For the Mean $+2 \mathrm{SD}_{\mathrm{Semi}}$ and Mean $+33 \mathrm{SD}_{\mathrm{Semi}}$ algorithms, a ROI was manually drawn within LV myocardium visually determined to be viable. Then, threshold values were defined as either $2 *$ SD above the mean image intensity of viable myocardium (for the Mean $+2 \mathrm{SD}_{\mathrm{Semi}}$ algorithm), as described previously [7, 11-13], or $3 * \mathrm{SD}$ above the mean image intensity of viable myocardium (for the Mean + $3 \mathrm{SD}_{\text {Semi }}$ algorithm), also described previously $[8,14]$.

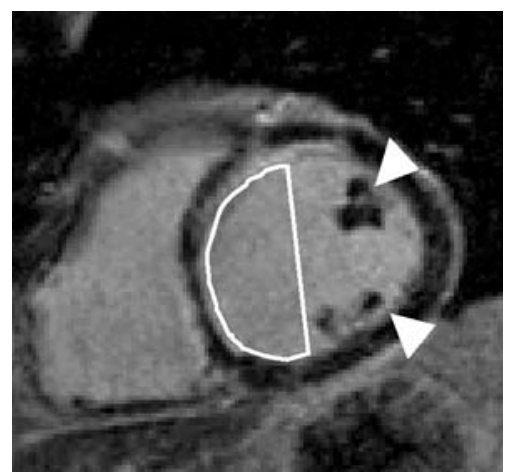

Figure 1. Basal short-axis image from patient 12, with papillary muscles evident near the anterolateral and inferolateral LV walls (arrows). For the Mean - 2SD $\mathrm{BP}$ algorithm, a ROI was selected in the LV blood pool (as shown); the ROI was restricted to the septal half to exclude the influence of papillary muscles on image intensity statistics.
For the Mean $+2 \mathrm{SD}_{\text {Auto }}$ and Mean $+3 \mathrm{SD}_{\text {Auto }}$ algorithms, an ROI was automatically specified by locating myocardial pixel(s) with the lowest image intensity and using it as the center of a $7 \times 7$ pixel region. Any pixels within this ROI but outside the LV myocardium were excluded. Then, the threshold was defined in a 2-step process. First, a preliminary threshold was defined as two times the standard deviation above the mean $\left(\right.$ Mean $\left.+2 \mathrm{SD}_{\text {Auto }}\right)$ or three times the standard deviation above the mean $\left(\mathrm{Mean}+3 \mathrm{SD}_{\text {Auto }}\right)$ of pixels within the initial ROI. A final threshold was then determined by removing any pixels within the ROI above the preliminary threshold and repeating the threshold calculation. This second step was an attempt to avoid including non-viable pixels, which might occur near the lowest intensity pixel.

The Minimum Intensity algorithm assumed that the histogram of all pixels within the LV epicardium (including blood pool) was bimodal, with peaks at the mean intensity of viable myocardium and the mean intensity of the LV blood pool; these groups are the two largest pixel types included in this histogram. A second-order polynomial curve was fit to all points in the histogram between these peaks and the intensity associated with the polynomial minimum was defined as the threshold (Figure 2).

\section{Data analysis}

After thresholding by each technique, the relative area of myocardial scar (Percent Scar) was determined as the ratio of non-viable myocardial pixels to total myocardial pixels within each slice. Isolated non-viable pixels, i.e. those without neighboring non-viable pixels, were excluded from the numerator in this calculation to reduce the influence of noise.

\section{Statistical analysis}

In all analyses, manual thresholding was used as the reference standard for evaluation of the remaining six thresholding algorithms. Paired Student's $t$-tests were used to assess the significance of differences between each of the six algorithms and the results from manual thresholding. 

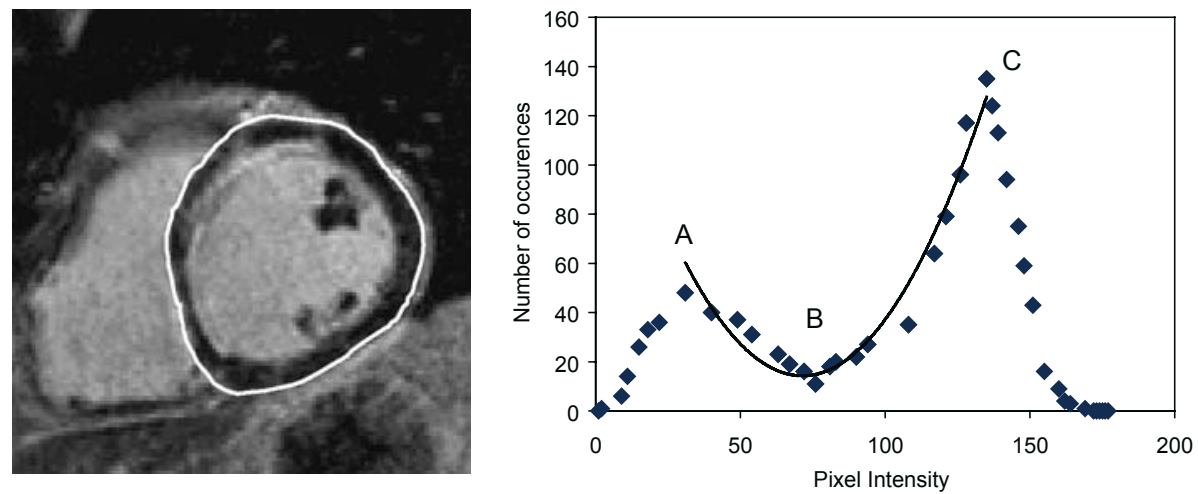

Figure 2. Summary of steps for thresholding by Minimum Intensity algorithm. (Left) Representative basal short-axis image (patient 12), including a manually drawn contour encompassing the LV myocardium and blood pool. (Right) Histogram of all pixels within the LV epicardial contour from the image at left. Also shown is a second order polynomial fit to all data between the LV myocardium peak (A) and LV blood pool peak (C). The minimum of this polynomial (B) was defined as the threshold for differentiating viable from non-viable myocardium.

Also, Bland-Altman analysis was performed to assess agreement between each algorithm and manually thresholded results [17]. Unpaired Student's $t$-tests were used to assess the significance of differences between pulse sequences. Oneway analysis of variance was used to test the significance of differences between short-axis levels. $p$-Values less than 0.05 were considered statistically significant.

\section{Results}

The mean ejection fraction was $28 \pm 11 \%$ (mean $\pm \mathrm{SD}$ ) (range 12-56\%), reflecting globally depressed cardiac function, on average, in these patients. The mean Percent Scar obtained from manual thresholding was $25 \pm 16 \%$ per slice overall, with significantly greater scar at the apex $(35 \pm 19 \%)$ than at the base $(18 \pm 11 \%$, $p<0.01)$ or mid-ventricle $(21 \pm 13 \%, p<0.01)$.

Histograms of viable myocardium, non-viable myocardium and LV blood pool were derived from manually thresholded images in each patient. There was no statistically significant difference in blood pool signal intensity between levels $(p=0.82)$. Overall, there was no statistically significant difference between the distributions of non-viable tissue pixels $(92 \pm 14)$ and LV blood pool pixels
$(90 \pm 9)$. However, the distribution of viable tissue pixels $(42 \pm 10)$ was significantly lower than that of both the non-viable tissue pixels and blood pool tissue pixels ( $p<0.01$ for each). Despite the clear separation in mean image intensity between viable and non-viable tissue, histograms of LV myocardium were rarely bimodal, displaying a clear nonviable tissue peak in 37 of 69 images (54\%), due to the small percentage of non-viable pixels relative to total myocardial pixels.

The distributions of image intensity in viable and non-viable myocardium, as well as in LV blood pool, for each patient, averaged over all three short-axis levels, are summarized in Table 1. Although comparisons of these image intensity values between patients has little meaning, because image intensity is measured in arbitrary units, Table 1 clearly demonstrates the overlap in image intensity between blood pool and non-viable myocardium in each patient, as well as the difference between these distributions and that of viable myocardium.

Table 2 shows Percent Scar for each algorithm averaged over all slices; the standard deviation of these results reflects variability between patients. Also in Table 2, the deviation from manually thresholded results is expressed as the mean difference (bias) and standard deviation of the difference (SD). Overall, the results from the Mean-SD ${ }_{\mathrm{BP}}$, 
Table 1. Image intensity for viable myocardium, non-viable myocardium and LV blood pool, averaged over all short-axis slices in each patient.

\begin{tabular}{cccc}
\hline Patient & Viable & Non-viable & Blood pool \\
\hline 1 & $33(11)$ & $93(18)$ & $85(20)$ \\
2 & $45(10)$ & $91(20)$ & $85(16)$ \\
3 & $7(4)$ & $21(6)$ & $23(5)$ \\
4 & $20(9)$ & $62(15)$ & $80(17)$ \\
5 & $30(15)$ & $106(27)$ & $94(19)$ \\
6 & $14(8)$ & $49(18)$ & $53(17)$ \\
7 & $79(16)$ & $142(19)$ & $147(31)$ \\
8 & $22(12)$ & $121(40)$ & $142(65)$ \\
9 & $27(12)$ & $129(19)$ & $133(22)$ \\
10 & $20(11)$ & $103(22)$ & $99(31)$ \\
11 & $46(19)$ & $77(20)$ & $126(41)$ \\
12 & $20(11)$ & $99(31)$ & $61(22)$ \\
13 & $46(19)$ & $114(25)$ & $136(32)$ \\
14 & $46(5)$ & $65(7)$ & $66(9)$ \\
15 & $53(10)$ & $82(8)$ & $84(11)$ \\
16 & $54(11)$ & $117(21)$ & $123(27)$ \\
17 & $10(6)$ & $40(14)$ & $26(10)$ \\
18 & $41(14)$ & $102(22)$ & $109(30)$ \\
19 & $20(9)$ & $61(14)$ & $46(11)$ \\
20 & $74(9)$ & $107(14)$ & $115(19)$ \\
21 & $54(7)$ & $-(-)^{\mathrm{a}}$ & $81(13)$ \\
22 & $100(7)$ & $125(8)$ & $118(9)$ \\
23 & $72(4)$ & $89(7)$ & $92(8)$ \\
\hline
\end{tabular}

These data are intended to highlight image intensity differences within patients, not for comparison between patients.

Results are mean (standard deviation).

aPatient had no non-viable tissue by manual thresholding.

Mean $+2 \mathrm{SD}_{\text {Semi }}$, Mean $+3 \mathrm{SD}_{\text {Auto }}$, and Minimum Intensity algorithms did not differ significantly from manual thresholding.

Results of Bland-Altman analysis are displayed in Table 2 and Figure 3. The mean bias (for all levels combined) was within $\pm 3 \%$ for five of the six algorithms, but was $10 \%$ for the Mean $+2 \mathrm{SD}_{\text {Auto }}$ algorithm. Furthermore, the limits of agreement were smallest for the two semi-automatic algorithms based on viable myocardium histograms $\left(\right.$ Mean $+2 \mathrm{SD}_{\text {Semi }}$, Mean $\left.+3 \mathrm{SD}_{\text {Semi }}\right)$, as seen in Figure 3. However, the limits of agreement were relatively large for the Mean-2 $\mathrm{SD}_{\mathrm{BP}}$ algorithm $( \pm 30 \%)$, Mean $+3 \mathrm{SD}_{\text {Auto }}( \pm 32 \%)$ algorithm, and the Minimum Intensity $( \pm 24 \%)$ algorithm although the results from these algorithms were not significantly different from the manual results.
Manual thresholding resulted in Percent Scar of $25 \pm 15 \%$ for the IR sequence and $24 \pm 17 \%$ for the PS sequence $(p=0.83)$. Results for each of the 6 algorithms, separated by pulse sequence, are shown in Table 3 . In general, results were consistent between sequences. Both the Mean $+3 \mathrm{SD}_{\text {Semi }}$ and the Mean $+2 \mathrm{SD}_{\text {Auto }}$ algorithms differed significantly from manual thresholding in both the IR and PS techniques. None of the remaining algorithms showed a significant difference from manual thresholding for either pulse sequence.

\section{Discussion}

In this study, we have described and evaluated six algorithms for segmentation of non-viable myocardium in DE-MRI images. Evaluation was accomplished by comparing results from each technique with results from manual thresholding, which has recently been established as a reliable technique for segmentation of DE images [10], and serves as the de facto gold standard for scar quantification in the absence of direct histological correlation.

In general, the six algorithms analyzed in this study demonstrated low mean bias compared to manual thresholding, with four producing results that were not significantly different from the manual results. However, the limits of agreement were generally large (see Figure 3), indicating that some algorithms would require user intervention to manually correct results in some patients. Furthermore, the algorithms were found to perform comparably in IR and PS images, important because they should be insensitive to pulse sequence if they are to be applied clinically.

Five of the 69 slices analyzed $(7 \%)$ had $\geq 50 \%$ scar by manual thresholding; all of these were apical slices. Based on these limited data, the bias in Percent Scar tended to be greater for slices with $\geq 50 \%$ scar, than for those with $<50 \%$ scar, independent of the algorithm used. However, confidence intervals were comparatively large. Because of this, only the Mean $+3 \mathrm{SD}_{\mathrm{Semi}}$ algorithm had a bias significantly different than zero in these slices. 
Table 2. Summary of results for each algorithm, separated by level and with all levels combined. Also shown is the mean difference (bias) between each algorithm and manual thresholding, and the results of paired $t$-test comparison with manual thresholding.

\begin{tabular}{|c|c|c|c|c|}
\hline Technique & Slice position & Percent Scar mean (SD) & Bias (SD) & $p$-Value \\
\hline \multirow[t]{4}{*}{ Mean-2SD ${ }_{\mathrm{BP}}$} & Apex & $30(19)$ & $-5(18)$ & 0.24 \\
\hline & Mid & $27(18)$ & $6(16)$ & 0.20 \\
\hline & Base & $23(14)$ & $5(11)$ & 0.16 \\
\hline & Combined & $25(17)$ & $1(15)$ & 0.59 \\
\hline \multirow[t]{4}{*}{ Mean $+2 \mathrm{SD}_{\mathrm{Semi}}$} & Apex & $32(17)$ & $-2(7)$ & 0.25 \\
\hline & Mid & $21(13)$ & $0(6)$ & 0.72 \\
\hline & Base & $19(11)$ & $1(6)$ & 0.17 \\
\hline & Combined & $25(15)$ & $0(6)$ & 0.84 \\
\hline \multirow[t]{4}{*}{ Mean $+3 \mathrm{SD}_{\mathrm{Semi}}$} & Apex & $30(16)$ & $-5(8)$ & $<0.01$ \\
\hline & Mid & $18(12)$ & $-3(6)$ & 0.06 \\
\hline & Base & $15(10)$ & $-2(6)$ & 0.07 \\
\hline & Combined & $21(14)$ & $-3(7)$ & $<0.01$ \\
\hline \multirow[t]{4}{*}{ Mean $+2 \mathrm{SD}_{\text {Auto }}$} & Apex & $38(20)$ & $3(21)$ & 0.78 \\
\hline & Mid & $33(18)$ & $11(11)$ & $<0.01$ \\
\hline & Base & $33(20)$ & $15(23)$ & $<0.01$ \\
\hline & Combined & $34(20)$ & $10(19)$ & $<0.01$ \\
\hline \multirow[t]{4}{*}{ Mean $+3 \mathrm{SD}_{\text {Auto }}$} & Apex & $28(15)$ & $-7(21)$ & 0.08 \\
\hline & Mid & $25(16)$ & 3 (12) & 0.11 \\
\hline & Base & $21(14)$ & $3(14)$ & 0.26 \\
\hline & Combined & $24(17)$ & $0(16)$ & 0.87 \\
\hline \multirow[t]{4}{*}{ Minimum intensity } & Apex & $36(22)$ & $1(17)$ & 0.18 \\
\hline & Mid & $25(18)$ & $4(10)$ & 0.05 \\
\hline & Base & $19(12)$ & $1(8)$ & 0.63 \\
\hline & Combined & $26(19)$ & $2(12)$ & 0.14 \\
\hline
\end{tabular}

\section{Manual thresholding technique}

The interobserver and intraobserver agreement for the manual thresholding technique have been evaluated previously [10], in a study which demonstrated no statistically significant differences in Percent Scar either between or within readers. Also, agreement by Bland-Altman analysis was good, with a bias of only $1 \%$ Percent Scar both between and within readers. Lastly, intraclass correlation coefficients of 0.84 for interobserver agreement and 0.88 for intraobserver agreement were found.

\section{Clinical application of algorithms}

Five of the six algorithms evaluated in this study were deemed suitable for clinical implementation (all but Mean $+2 \mathrm{SD}_{\text {Auto }}$ ); these algorithms can be divided into two categories based on their agreement with manual thresholding results.
Two algorithms, Mean $+2 \mathrm{SD}_{\text {Semi }}$ and Mean $+3 \mathrm{SD}_{\text {Semi }}$, proved to be suitable for direct clinical implementation, as evidenced by their near-zero bias and consistently low limits of agreement at all short-axis levels. These algorithms have been described and implemented in several previous studies [7, 8, 11-14]. Although the Mean $+3 S_{\text {Semi }}$ algorithm did differ significantly from the manual technique, Percent Scar differed by $<10 \%$ in $80 \%$ (55 of 69 ) of images using this technique, with an overall bias of only $-3 \%$.

Three algorithms (Mean-2SD ${ }_{\mathrm{BP}}, \quad$ Mean + $3 \mathrm{SD}_{\text {Auto }}$, Minimum Intensity) proved reliable on average. However, although these algorithms did not differ significantly from manual thresholding, and demonstrated low bias, their limits of agreement were large $( \pm 24$ to $\pm 32 \%)$. Thus, they are deemed suitable only for initial estimates of scar extent in individual patients, but any software implementing them should contain tools for manual correction before results can be applied clinically. 

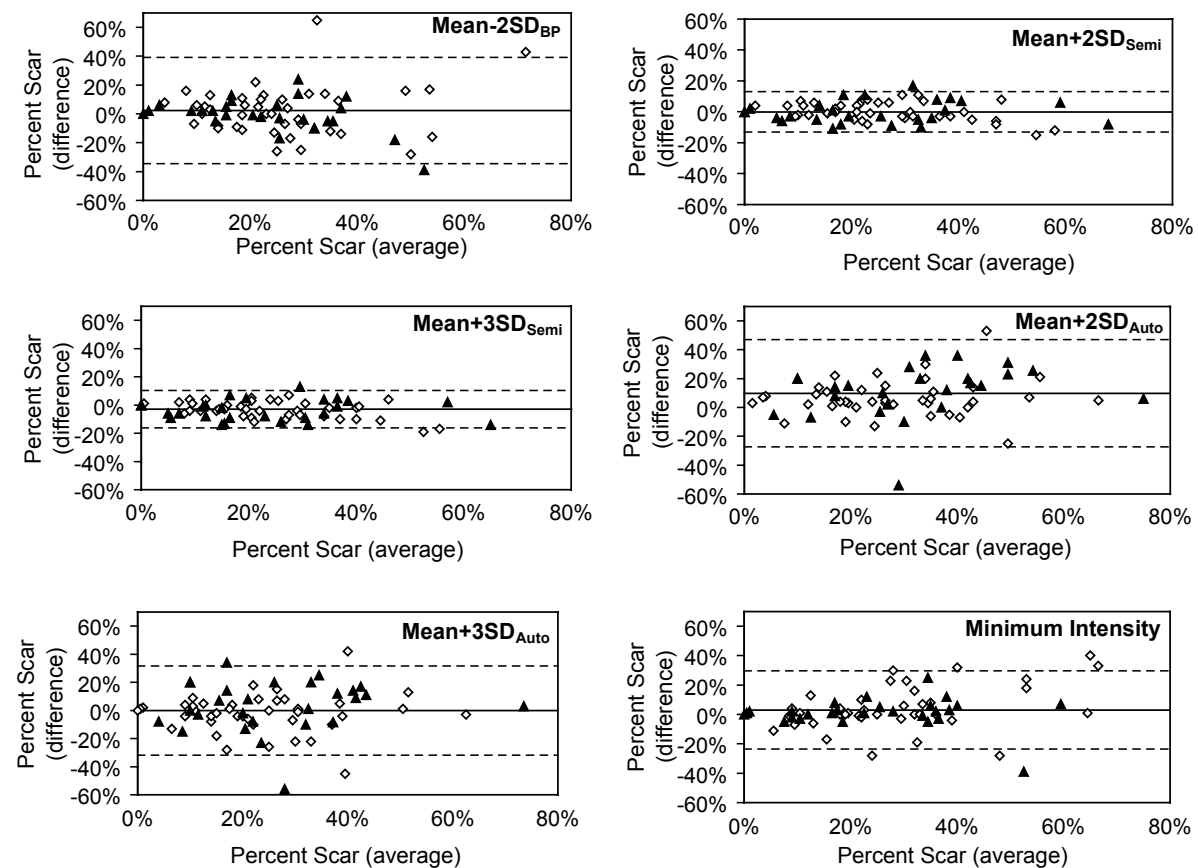

Figure 3. Plots of agreement between each algorithm and manual thresholding results, by Bland-Altman analysis (17). Solid lines show the mean bias for each algorithm; dashed lines are limits of agreement. Individual data points are separated by pulse sequence $(\mathbf{\Delta} P S$ $\diamond \mathrm{IR})$.

A shortcoming of algorithms in the first category (i.e. Mean $+2 \mathrm{SD}_{\text {Semi }}$, Mean $+3 \mathrm{SD}_{\text {Semi }}$ ) is that they require users to manually draw a ROI in viable myocardium, from which the threshold is derived. In contrast, algorithms in the second category permit fully automatic implementation (after the LV myocardium has been segmented).

Table 3. Influence of imaging sequence on thresholding algorithms, showing mean Percent Scar, bias and results of paired $t$-test between each algorithm and manual thresholding.

\begin{tabular}{|c|c|c|c|c|}
\hline Sequence & Technique & Percent Scar Mean (SD) & Bias (SD) & $p$-Value \\
\hline \multirow[t]{6}{*}{ Inversion recovery } & Mean $-2 \mathrm{SD}_{\mathrm{BP}}$ & $27(18)$ & $2(18)$ & 0.53 \\
\hline & Mean $+2 \mathrm{SD}_{\mathrm{Semi}}$ & $25(13)$ & $0(6)$ & 0.81 \\
\hline & Mean $+3 \mathrm{SD}_{\mathrm{Semi}}$ & $22(13)$ & $-3(6)$ & $<0.01$ \\
\hline & Mean $+2 \mathrm{SD}_{\text {Auto }}$ & $31(17)$ & $7(13)$ & $<0.01$ \\
\hline & Mean +3 SD $_{\text {Auto }}$ & $22(16)$ & $0(14)$ & 0.31 \\
\hline & Minimum Intensity & $28(21)$ & $0(14)$ & 0.14 \\
\hline \multirow[t]{6}{*}{ Phase sensitive } & Mean $-2 \mathrm{SD}_{\mathrm{BP}}$ & $23(12)$ & $1(12)$ & 0.76 \\
\hline & Mean +2 SD $_{\text {Semi }}$ & $24(18)$ & $0(7)$ & 0.96 \\
\hline & Mean $+3 S_{\text {Semi }}$ & $20(17)$ & $3(7)$ & 0.02 \\
\hline & Mean $+2 \mathrm{SD}_{\text {Auto }}$ & $39(23)$ & $15(25)$ & $<0.01$ \\
\hline & Mean $+3 \mathrm{SD}_{\text {Auto }}$ & $28(18)$ & $0(18)$ & 0.36 \\
\hline & Minimum intensity & $25(16)$ & $1(10)$ & 0.52 \\
\hline
\end{tabular}

Results are averaged over all three short-axis levels. 


\section{Algorithm assumptions}

Each of the algorithms used in this study rely on several assumptions about the shape of the histograms upon which they are based. For instance, the four myocardium based algorithms $\left(\right.$ Mean $+2 \mathrm{SD}_{\mathrm{Semi}}, \quad$ Mean $+3 \mathrm{SD}_{\mathrm{Semi}}, \quad$ Mean + $2 \mathrm{SD}_{\text {Auto }}$, Mean $+3 \mathrm{SD}_{\text {Auto }}$ ) rely on a normal distribution of pixels within viable myocardium, which was found to be true in all patients. Based on histograms derived from manual thresholding, in viable myocardium $69 \pm 8 \%$ of pixels were within one standard deviation of the mean image intensity, and $97 \pm 2 \%$ of pixels were within two standard deviations of the mean image intensity, consistent with the definition of a normal distribution (i.e., approximately $67 \%$ within one standard deviation of the mean and approximately $95 \%$ within two standard deviations of the mean) [18]. However, the ultimate success of the algorithms utilizing viable myocardium histograms depend upon a representative sampling of pixels within the user or automatically selected ROI.

For the Mean $+2 \mathrm{SD}_{\text {Auto }}$ and Mean $+3 \mathrm{SD}_{\text {Auto }}$ algorithms, automatic ROI selection was centered on the lowest intensity pixel in the myocardium, which did not always result in a representative sample of pixels from viable myocardium, and led to large limits of agreement. The mean area of the automatically determined ROI was 105 pixels at the base (range 17-372), 78 pixels at mid-ventricle (range 16-285) and 52 pixels at the apex (range 18-219). These areas exceed 49 pixels because many patients had multiple occurrences of the minimum pixel intensity value. However, the ROI area was often less than 49 pixels. In some cases, this resulted from thinning of the LV wall post-infarction; other times it was because the minimum intensity pixel was located near the endocardial or epicardial borders. Despite the variation in ROI area between patients, there was no statistically significant relationship between bias and ROI area for either automatic algorithm: Mean $+2 \mathrm{SD}_{\text {Auto }}$, BIAS $=$ 0.11-0.0002*AREA, $\mathrm{R}^{2}=0.005$; Mean $+3 \mathrm{SD}_{\text {Auto }}$, BIAS $=0.11-0.0003 * A R E A, R^{2}=0.017$ (in neither case was the slope of the regression line significantly different from zero).
The Mean-2SD $\mathrm{BP}_{\mathrm{BP}}$ algorithm was based on an assumed normal distribution of pixels within its blood pool ROI, an assumption which proved true on average, with $94 \pm 1 \%$ of blood pool pixels within two standard deviations of the mean. Lastly, the Minimum Intensity algorithm was dependent upon a bimodal distribution of myocardial and blood pool pixels, with a clear separation of peaks, which, by visual inspection, proved true in 61 of 69 images considered $(88 \%)$. Both the Mean-2SD $\mathrm{SP}_{\mathrm{BP}}$ and the Minimum Intensity algorithms also assumed that the histograms of non-viable tissue and blood pool would not differ significantly, which did prove valid.

\section{Conclusions}

In conclusion, this study has demonstrated that relatively simple algorithms, based on characteristic features of the histograms of LV myocardium and LV blood pool from DE-MRI images, have sufficient accuracy for clinical quantification of myocardial scar extent. However, due to the range of image quality possible in clinical imaging, as well as the presence of microvascular obstruction [19] or non-hyperenhancing scar [10], automatic algorithms could lead to erroneous scar extent estimates in some patients. Therefore, any software for quantification of non-viable myocardium in DE images should include manual correction capabilities to account for the small percentage of images in which these algorithms might fail.

\section{References}

1. Stillman AE, Wilke N, Jerosch-Herold M. Myocardial viability. Radiol Clin North Am 1999; 37: 361-378.

2. Bax JJ, de Roos A, van der Wall EE. Assessment of myocardial viability by MRI. J Magn Reson Imaging 1999; 10 : 418-422.

3. Choi KM, Kim RJ, Gubernikoff G, Vargas JD, Parker M, Judd RM. Transmural extent of acute myocardial infarction predicts long-term improvement in contractile function. Circulation 2001; 104: 1101-1107.

4. Kim RJ, Wu E, Rafael A, et al. The use of contrast-enhanced MRI to identify reversible myocardial dysfunction. New Engl J Med 2000; 343: 1445-1453. 
5. Wu E, Judd RM, Vargas JD, Klocke FJ, Bonow RO, Kim RJ. Visualisation of presence, location, and transmural extent of healed Q-wave and non-Q-wave myocardial infarction. Lancet 2001; 357: 21-28.

6. Klein C, Nekolla SG, Bengel FM, et al. Assessment of myocardial viability with contrast-enhanced magnetic resonance imaging: comparison with positron emission tomography. Circulation 2002; 105: 162-167.

7. Kim RJ, Fieno DS, Parrish TB, et al. Relationship of MRI delayed contrast enhancement to irreversible injury, infarct age and contractile function. Circulation 1999; 100: 19922002.

8. Fieno DS, Kim RJ, Chen EL, Lomasney JW, Klocke FJ, Judd RM. Contrast-enhanced magnetic resonance imaging of myocardium at risk. J Am Coll Cardiol 2000; 36: 19851991.

9. Sandstede JJW, Lipke C, Beer M, et al. Analysis of first-pass and delayed contrast-enhancement patterns of dysfunctional myocardium on MR imaging: use in the prediction of myocardial viability. AJR 2000; 174: 1737-1740.

10. Setser RM, Bexell DG, O'Donnell TP, et al. Quantitative assessment of myocardial scar in delayed-enhancement magnetic resonance imaging. J Magn Reson Imaging 2003; 18: 434-441.

11. Gerber BL, Rochitte CE, Bluemke DA, et al. Relation between Gd-DTPA contrast enhancement and regional inotropic response in the periphery and center of myocardial infarction. Circulation 2001; 104: 998-1004.

12. Gerber BL, Garot J, Bluemke DA, Wu KC, Lima JAC. Accuracy of contrast-enhanced magnetic resonance imaging in predicting improvement of regional myocardial function in patients after acute myocardial infarction. Circulation 2002; 106: 1083-1089.
13. Marholdt H, Wagner A, Holly TA, et al. Reproducibility of chronic infarct size measurement by contrast-enhanced magnetic resonance imaging. Circulation 2002; 106: 2322 2327.

14. Hillenbrand HB, Kim RJ, Parker MA, Fieno DS, Judd RM. Early assessment of myocardial salvage by contrastenhanced magnetic resonance imaging. Circulation 2002; 102: 1678-1683.

15. Simonetti OP, Kim RJ, Fieno DS, et al. An improved MR imaging technique for the visualization of myocardial infarction. Radiology 2001; 218: 215-223.

16. Kellman P, Arai AE, McVeigh ER, Aletras A. Phase-sensitive inversion recovery for detecting myocardial infarction using gadolinium delayed hyperenhancement. Magn Reson Med 2002; 47: 372-383.

17. Bland JM, Altman DG. Statistical methods for assessing agreement between two methods of clinical measurement. Lancet 1986; 1: 307-310.

18. Samuels ML, Witmer JA. The normal distribution. In: Statistics for the Life Sciences. Prentice Hall, 1999; 500-559.

19. Gerber BL, Rochitte CE, Melin JA, et al. Microvascular obstruction and left ventricular remodeling early after acute myocardial infarction. Circulation 2000; 101: 2734-2741. 